Clinical microbiology

\title{
Anaerobes and laboratory automation: Like oil and water?
}

\author{
Márió Gajdács ${ }^{\mathrm{a}, \mathrm{b}, *}$ \\ a Department of Pharmacodynamics and Biopharmacy, Faculty of Pharmacy, University of Szeged, Eötvös utca 6., 6720, Szeged, Hungary \\ ${ }^{\mathrm{b}}$ Institute of Clinical Microbiology, Faculty of Medicine, University of Szeged, 6725, Szeged, Semmelweis utca 6., Hungary
}

\section{A R T I C L E IN F O}

\section{Article history:}

Received 7 May 2019

Received in revised form 13 June 2019

Accepted 19 June 2019

Available online $\mathrm{xxx}$

Handling Editor: Audrey Schuetz

Keywords:

Anaerobes

Total laboratory automation

TLA

Clinical microbiology

Advantages

Limitations

Perspectives

MALDI-TOF MS

\begin{abstract}
A B S T R A C T
Diagnostic laboratories are urged to take advantage of novel technological advancements to provide standardized and high-throughput information for clinicians; however, total laboratory automation (TLA) has only recently been introduced in clinical microbiology in the last 10-12 years. The introduction of total laboratory automation comes with certain advantages and drawbacks that need to be assessed before the introduction of such systems in the diagnostic workflow that includes the detection of anaerobic bacteria. For several reasons, there is yet to be a manufacturer to fully address the issue of anaerobes in the setting of laboratory automation; the aim of the present paper is to address some of the issues associated with anaerobes in lab automation.
\end{abstract}

(C) 2019
Due to the increasing costs and continuous demand for efficacy improvement by governments and relevant stakeholders worldwide, healthcare institutions are urged to remain competitive and to take advantage of novel technological advancements. Diagnostic laboratories, having a pivotal role in patient care and outcomes, are facing the demand of effective and high-quality services for the lowest possible price [1]. The process of automation in medical laboratories has been a steadily increasing trend, including both research and diagnostic laboratories, such as clinical chemistry, hematology and molecular biology; however, it has only been recently introduced in clinical microbiology in the last 10-12 years [2]. Although several automated inoculators have been introduced to the market, currently only two manufacturers (Copan and BD Kiestra) offer instrumentation and services for total laboratory automation (TLA) [3]. The potential advantages of laboratory automation include: savings in personnel costs, a pronounced increase in productivity and reproducibility, connection with the laboratory information system (LIS), matrix-assisted laser desorption/ionization time-of-flight mass spectrometry (MALDI-TOF) or antimicrobial susceptibility testing (AST) instruments, additional safety for the labor force (both from pathogens and

ergonomic

\footnotetext{
* Department of Pharmacodynamics and Biopharmacy, Faculty of Pharmacy, University of Szeged, Eötvös utca 6., 6720, Szeged, Hungary.

Email address: mariopharma92@gmail.com (M. Gajdács)
}

injuries; i.e. due to repeated motion, awkward and sustained poses), the possibility to introduce telemicrobiology services (if the demand arises), the cost of consumables is relatively constant; therefore, they can be planned in advance (i.e. in an institutional budget), and it may also result in significant cost reduction, after the return on investment (ROI) time has elapsed (which is characteristic to each individual setting) [1-3]. However, there are also drawbacks that must be considered: the initial costs of the instrumentation and the following maintenance costs are very high, coupled by the general expectation of increased production by hospital management, which is usually not feasible in the initial period of introduction; backup methods must be available in case of a system malfunction and there has to be significant adaptation in the sample processing workflow and training of the staff in the laboratory. If the latter is not achieved (logistical issues with sample transport and processing, inadequate training or resistance from the staff, inappropriate or no adjustments in the working hours of the institution etc.), the turnaround time (TAT) for results may actually become longer, compared to the conventional laboratory workflow. In addition, several sample types (tissue samples, aspirates) and pathogens (strict anaerobes, fungi) have remained on the periphery, when it comes to TLA [1-3].

Until recently, the cultivation and identification of strict anaerobic bacteria has mostly been a neglected topic of interest in most clinical microbiology laboratories, due to the laborious nature of the isolation, species-level identification and predictable resistance patterns [4]. However, because these bacteria have been described in infec- 
tions in almost all anatomical sites (and due to the surge of interest regarding their roles in the human microbiome), nowadays more laboratories invest resources in the correct identification (aided by MALDI-TOF MS or 16S rRNA gene sequencing) and susceptibility testing of these pathogens $[5,6]$. Despite the promising developments in rapid ID/AST technologies and surge in interest regarding these microorganisms, there is yet to be a manufacturer to fully address the issue of anaerobes in the setting of laboratory automation. The features of anaerobic bacteriology in the clinical setting makes its integration in the TLA workflow difficult, which is unfortunate, because the added standardization and reproducibility in their inoculation, identification and AST would be greatly appreciated. In fact, there are reports on the rapid (4-8h) identification of anaerobes from blood cultures (allowing for the supply of information in a clinically relevant time frame) and on the detection of various resistance mechanisms/typing using MALDI-TOF MS technology [7,8]. These novel modalities in the clinical microbiology laboratories could complement automation at the point of reading the culture, and in the following steps, such as AST or typing.

Thus far, the cultivation of anaerobes has remained external to lab automation, as the main problem is that incubators cannot provide an anaerobic atmosphere at the moment. Nevertheless, there is an option of incubating the plates in appropriate external anaerobic systems/incubators, but this breaks the continuity providing anaerobic atmosphere. After that, these plates may be inserted into the plate imaging/screening system for image analysis and subsequent picking of colonies for further processing [3]. In automated systems, plates are primarily analyzed based on the pictures of the plates available in the user interface, with the option of "calling out" the plates for organoleptic evaluation. Anaerobic pathogens have a characteristic odor, which is extremely useful in determining their presence during presumptive identification. Unfortunately, without calling out the plates, there is no way to ascertain the smell of the colonies. Nevertheless, the plates spend a significant amount of time in atmospheric oxygen during their way through the different workstations of the system (especially if this process is repeated several times), which may be detrimental to some bacteria that are particularly sensitive to oxygen (e.g., Fusobacterium spp., Solobacterium moorei) [9]. An oxygen-shock of 15-30 min may be lethal to the strict anaerobes (those not possessing any superoxide-dismutase or catalase enzymes), both directly after the inoculation of plates and after the colonies are already present on the culture media.

An advantage of automated systems is the consistent way of streaking, which additionally allows for consistent, comparable and reliable results (i.e. isolated colonies), even from samples with low colony counts. However, there is also the concern regarding the picking of colonies for analysis using the electronic interface. In addition, some samples cannot be incorporated in the preanalytical automated systems aiming to provide a liquid sample (which is the pre-requisite for plating automation); therefore, these samples have to be processed in partially automated system, additionally complicating laboratory logistics [3]. These samples are usually considered "precious" (e.g., surgical samples, tissue samples acquired by biopsy, abscesses, aspirates), and coincidentally, the clinical relevance of the isolation of anaerobes in these sample types is high.

Anaerobes predominantly occur in mixed infections and the size of their colonies are much smaller, compared to facultative anaerobes; although in some cases, the color of their colonies may aid visual interpretation (depending on the culture media). Adequate image quality and careful selection are needed to process the pathogens of importance further. However, the users may actually lose pinpoint colonies during the process of imaging, where a pixel problem could mask colonies and mislead the microbiologists. The same issue may plague the processing of polymicrobial samples: without the adequate image quality, the separation of (which frequently grow on top of each other) is close to impossible (or depends on the expertise/experience of the laboratory assistants). As some anaerobes produce fluorescence under ultraviolet light (which is a useful feature during presumptive identification), a UV-panel may aid colony picking in mixed (aerobic-anaerobic) cultures and for small colonies [10].

There are a number of potential ways to aid the inclusion of anaerobes in an automated system. The introduction of a dedicated anaerobic incubator system (chamber) in the workflow of the existing automated systems could be a possible solution. This chamber should be as close as possible to the site of medium inoculation, to prevent the damaging effects of oxygen against these microorganisms. If manageable, the development of a "fast lane" (which would have the ability to rapidly transfer anaerobic plates only in the shortest possible time) or a sealed, anaerobic pathway on the carousel (from the inoculation site to the chamber) would ensure the viability of these pathogens. Anaerobic culture media could be differentiated by the machine with the use of barcoding thus, these plates would automatically be transferred separately from aerobic plates on the carousel. Another welcome addition would be the installation of cameras inside the anaerobic chamber, to allow for the inspection of individual plates without the need to remove them from anaerobic environment. This way, only those plates would need to be exposed to oxygen, where further processing (colony picking for MALDI-TOF or AST) is indicated. The improvements in optical technologies and image processing (resulting in sharper images) Although this technology is still in the experimental phases of development, so-called "electronic noses" (or e-sensing devices) may be utilized in these systems for the detection of short-chain fatty acids produced by these microorganisms, obviating the need to actually smell the plates.

In summary, laboratory automation gives the opportunity to clinical microbiology laboratories to take advantage of current technological advancements to provide standardized and high-throughput information for clinicians and by proxy, care for the patients. Strict anaerobes are a group of pathogens, where there are still concerns and issues not addressed by companies, mainly due to their biological properties; however this may be influenced by the still existing disinterest in their cultivation in some laboratories; therefore, maybe the need is just not present yet. In addition, the differences in sample volumes (e.g., urine, respiratory and fecal samples arrive to clinical microbiology in much larger numbers and they are already in liquid form, ready for processing) may also have an influence, if observed from a financial perspective $[1-3,11,12]$. With the description of anaerobes in various infectious processes in increasing amount, developers should soon reconsider this area as a potential field of future development.

\section{Abbreviation}

AST: antimicrobial susceptibility testing; ID: identification; LIS: laboratory information system; MALDI-TOF MS: matrix-assisted laser desorption/ionization time-of-flight mass spectrometry; ROI: return on investment; TAT: turnaround time; TLA: total laboratory automation; $\mathbf{U V}$ : ultraviolet.

\section{Conflicts of interest}

The author declares no conflict of interest, monetary or otherwise. 


\section{Acknowledgements}

M.G. was supported by the National Youth Excellence Scholarship [Grant Number NTP-NTFÖ-18-C-0225] and the ESCMID Mentorship and Observership Programme.

\section{References}

[1] C. Archetti, A. Montaneli, D. Finazzi, L. Caimi, E. Garaffa, Clinical laboratory automation: a case study, J. Public Health Res. 6 (2017) 881.

[2] S.M. Novak, E.M. Marlowe, Automation in the clinical microbiology laboratory, Clin. Lab. Med. 33 (2013) 567-588.

[3] A. Croxatto, G. Prod'hom, F. Faverjon, Y. Rochais, G. Greub, Laboratory automation in clinical bacteriology: what system to choose?, Clin. Microbiol. Infect. 22 (2016) 217-235.

[4] M. Gajdács, G. Spengler, E. Urbán, Identification and antimicrobial susceptibility testing of anaerobic bacteria: Rubik's cube of clinical microbiology?, Antibiotics 6 (2017) e25.

[5] E. Nagy, L. Boyanova, U.S. Justesen. on behalf of ESGAI. How to isolate, identify and determine antimicrobial susceptibility ofanaerobic bacteria in routine laboratories?, Clin. Microbiol. Infect. 24 (2018) 1139-1148.
[6] A.C. Veloo, E.D. de Vries, H. Jean-Pierre, U.S. Justesen, T. Morris, E. Urbán, I. Wybo, A.J. van Winkelhoff, On behalf of the ENRIA workgroup. The optimization and validation of the Biotyper MALDI-TOF MS database for the identification of Gram-positive anaerobic cocci, Clin. Microbiol. Infect. 22 (2016) 793-798.

[7] S. Shannon, D. Kronemann, R. Patel, A.N. Schuetz, Routine use of MALDI-TOF MS for anaerobic bacterial identification in clinical microbiology, Anaerobe 54 (2018) 191-196.

[8] E. Nagy, S. Becker, J. Sóki, E. Urbán, M. Kostrzewa, Differentiation of division I (cfiA-negative) and division II (cfiA-positive) Bacteroides fragilis strains by matrix-assisted laser desorption/ionization time-of-flight mass spectrometry, J. Med. Microbiol. 60 (2011) 1584-1590.

[9] W.J. Loesche, Oxygen sensitivity of various anaerobic bacteria, Appl. Microbiol. 18 (1969) 723-727.

[10] J.S. Brazier, A note on ultra-violet red fluorescence of anaerobic bacteria in vitro, J. Appl. Microbiol. 60 (1982) 121-126.

[11] I. Bruckhardt, Laboratory automation in clinical microbiology, Bioengineering 5 (2018), e102.

[12] M. Gajdács, E. Urbán, Epidemiology and species distribution of anaerobic Gram-negative cocci: a 10-year retrospective survey (2008-2017), Acta Pharm. Hung. (2019), accepted. 